\title{
Intelligent Control and HCI for a Powered Wheelchair Using a Simple Expert System and Ultrasonic Sensors
}

\author{
David Sanders $^{1}$, Malik Haddad ${ }^{1(凶)}$, Peter Omoarebun ${ }^{1}$, Favour Ikwan ${ }^{1}$, \\ John Chiverton ${ }^{1}$, Shikun Zhou ${ }^{1}$, Ian Rogers ${ }^{2}$, and Boriana Vatchova ${ }^{3}$ \\ 1 University of Portsmouth, Portsmouth PO1 3DJ, UK \\ \{david.sanders, malik.haddad\}@port.ac.uk \\ 2 Gems Sensors \& Controls, Lennox Road, Basingstoke RG22 4AW, UK \\ ${ }^{3}$ Bulgarian Academy of Sciences, 1113 Sofia Akad.G.Bonchev Str., bl.2, Sofia, Bulgaria
}

\begin{abstract}
Intelligent control and human computer interaction is investigated for a powered wheelchair using a simple expert system and ultrasonic sensors. The aim is to make driving easier. Signals from sensors and joysticks are interpreted. The interpreted signals are mixed so that the systems collaborate with the human driver to improve their control over direction and speed. Ultrasonic sensors identify hazards and the system suggests a safer speed and direction. Results are presented from drivers completing a series of timed routes using joysticks to control wheelchairs both with a microcomputer and sensors assisting them and without. Recently published systems are used to contrast and compare results. The new system described in this paper consistently performed better. An additional result appears to be that the amount of support from the microcomputer and sensors should be altered depending on surroundings and situations. The research is part of a bigger research project to improve mobility and enhance the quality of life of disabled powered wheelchair users by increasing their self-reliance and self-confidence.
\end{abstract}

Keywords: Expert · System · Disabled · Smart · Wheelchair · Sensor · Ultrasonic

\section{Introduction}

Interest in enhanced quality of life is rising as modern medical treatment is improving survival rates and life expectancy is increasing [1]. Smart wheelchairs can help enhance that quality of life. They have sensors and work with cognitive techniques developed during research into mobile robots. But they tend not to act so autonomously. Instead they try to extend or complement the abilities of a disabled driver. For disabled and aging people who cannot walk, smart wheelchairs can provide some significant benefits and enhance their quality of life by maintaining their mobility, broadening (and continuing) social and community activities, conserving energy and strength. But because powered wheelchairs can be difficult to drive, some automation can be helpful [2]. George Klein 
invented the first powered wheelchair (with the National Research Council of Canada) to assist quadriplegics injured during World War Two [3].

Smart wheelchairs have often been thought of as mobile robots with an added seat but the difference between powered wheelchairs and robots is that the powered wheelchair becomes an extension of their human driver. The disabled driver sits on it and needs to be comfortable driving it (and sitting in it) [4]. Disability systems including intelligent powered wheelchairs need to accommodate many different types of disability.

The World Health Organization suggest that $15 \%$ of the population of the world live with a disability, and between $2 \%$ and $4 \%$ experience considerable problems in practice. Global estimates of disability are rising as the world population ages and improvements have been made to the assessment and measurement of disability [5].

This paper describes a simple expert system $[6,7]$ to control a powered wheelchair [8]. Ultrasonics [9] identify obstacles and propose more suitable and safer speeds and directions. Systems in use now [10-13] rely on driver experience and their ability to see. Work described in this paper interprets sensor and joystick signals and then uses an expert system to make driving easier for a disabled user.

The way humans interact might diminish effectiveness [10-15] and some ways of improving interaction are considered, especially if disturbance are present because of differences between wheels or because of hills, slopes and surfaces [16]. Algorithms mix data from a joystick with data from ultrasonic sensors. Drivers show a desired direction using a joystick and the wheelchair tends to move in that direction. Drivers react to disturbances and revise their desired direction if necessary. The intelligent system described in this paper, processed data from a joystick and from sensors and used the information from that to assist the disabled driver.

Powered wheelchairs have often been guided with a joystick [10-18] although there are other devices: switches [19], pointers [20, 21] or virtual reality transducers [22].

Drivers need to drive safely and avoid obstacles (people, wheelchairs, other wheelchairs or vehicles) [23,24]. Local sensing has been used with powered wheelchairs, for example laser or light [25], ultrasonics [10-18] or infra-red [26] and position sensing has used gyro, tilt, acoustic or odometry [27]. GPS [28] does not work well inside or when shielded but Assisted GPS [29] can be helpful. Vision [30-36] uses more processing power but as the cost of computing power has reduced [37] they have become more and more popular. The human driver still tends to provide the best data about the environment and what is to be achieved. Because they are cost effective, simple and robust [10-17, 38-40], ultrasonics were chosen for the work described here.

\section{The Wheelchair Systems}

Smart wheelchairs usually comprise of a standard powered wheelchair with sensors and a computer added, or a mobile robot base with a seat mounted on top. In this work a powered wheelchair with large driving wheels and trailing casters was used. Cameras could be attached to the front of the wheelchair in between the driving wheels. Pairs of ultrasonic transducers could be attached. Two above each driving wheel and a third in the center at the front. Receiver/transmitter pairs were attached at the front of the wheelchair $[8,14]$. They transmitted a one millisecond pulse of sound. That pulse reflected back 
from obstacles and then ranges were calculated from the times taken for pulses to return. In that way, any obstacles in front of the wheelchair could be sensed.

Links between joysticks and wheelchairs were disconnected. Instead, a microcomputer handled control in one of three modes:

- Ultrasonics interrogated by the microcomputer and it modifies the wheelchair bearing using either:

- The new algorithms.

- Algorithms published recently and used for comparison.

- Wheelchair driven directly by the Joystick.

The code structure is shown in Fig. 1. Rules were: apply smooth and controlled movements; user remains in overall control; only modify the trajectory of the wheelchair when necessary. An imaginary potential field was created around obstacles [36].

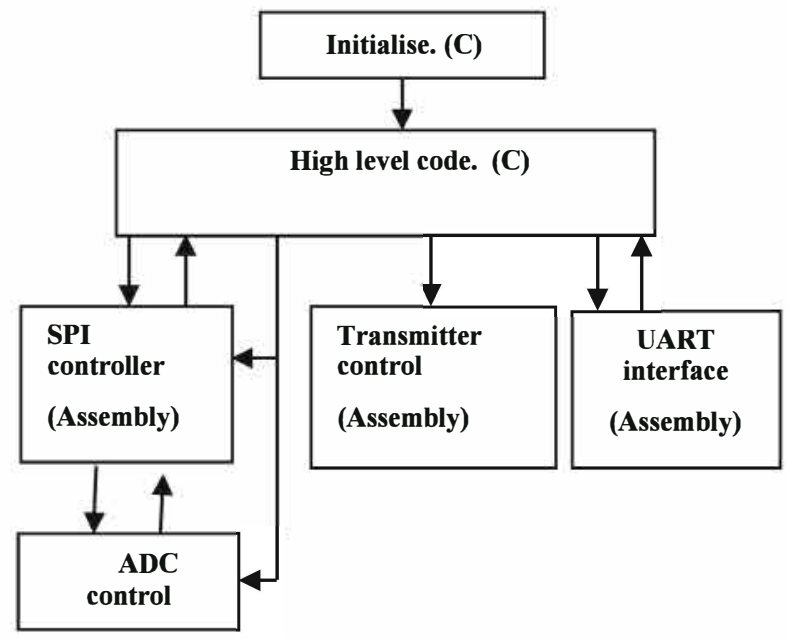

Fig. 1. Code structure.

Longer sound pulses contain more energy and can detect objects further away. Sound waves travel at approximately $330 \mathrm{~m} / \mathrm{s}$. If a pulse is three milliseconds long then the length of the pulse is about one meter. The minimum range is then half a meter if the pulse length is three milliseconds. Shorter ranges were required and lengths of 50 us, 100 us, 500 us, and $1 \mathrm{~ms}$ were considered. Pulse lengths were switched automatically by a "range finder". If the "range finder" did not detect anything then it incremented pulse lengths so that range extended.

Ultrasonics were relatively noisy and misreadings were filtered out. Volumes in front of the wheelchair were placed into an array of three sections: close, near and far away. If an object was detected then distance to the object was classified as: close, near or faraway. Ultrasonic transmitter/receiver pairs were attached to the front of the wheelchair so that their beams enclosed the volume in front of the wheelchair. The transducers are represented in Fig. 2. 


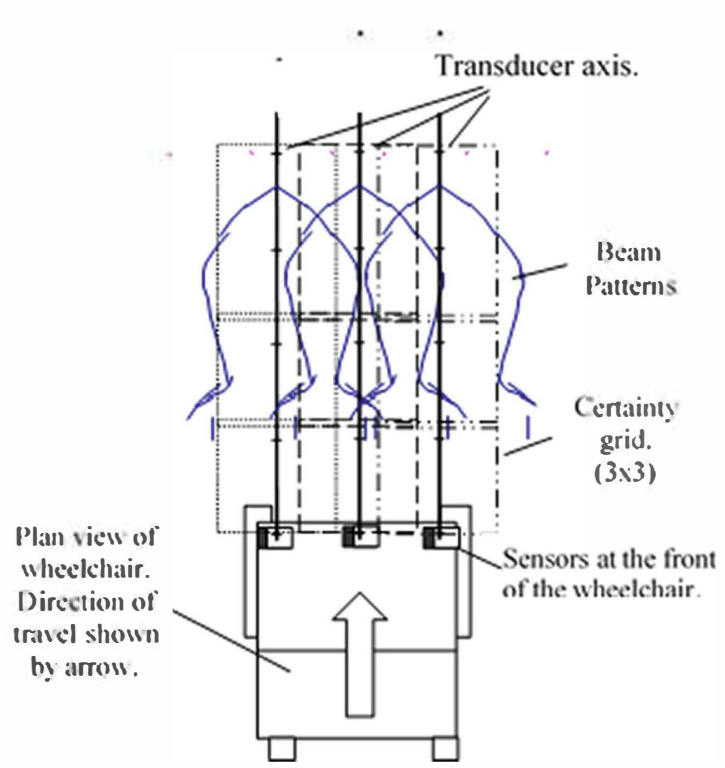

Fig. 2. Three sensors and the array representing the volume ahead.

Elements in the grid that contained an obstacle were increased by a high amount, for example, 5 . The other elements were incremented by a smaller number, for example, 1. Elements had a maximum value of fifteen and minimum value of zero.

Objects within an element of the grid prompted an increase in value within that element. A haphazard reading within other elements would increase them briefly, but those readings reduced every time the system updated. If an object relocated to another element then the old grid element reduced in value and the new element increased. Consistent measures of distance were acquired within half a second. Figure 3 shows the structure of the object detection process.

\section{Algorithms to Interpret the Joystick}

The Penny \& Giles Joystick on the wheelchair contained two potentiometers providing two voltages. The voltages denoted the position of the joystick. They were read by an Analogue to Digital Converter and then converted from Cartesian co-ordinates to Polar co-ordinates: $|\mathrm{J}| \angle \theta$. Where $|\mathrm{J}|$ represented desired speed and $\angle \theta$ represented desired direction. Standard $C$ libraries were used for mathematical functions.

Joystick magnitude and angle were calculated using:

$$
\text { argument }=\text { JS0/JS1; opposite/adjacent (ATAN) }
$$

bearing $=\operatorname{atan}(\operatorname{argument}) ;$ Angle of joystick in radians

$$
\text { Magnitude }=\operatorname{sqrt}((\mathrm{JS} 1 * \mathrm{JS})+(\mathrm{JS} 0 * \mathrm{JS} 0))
$$




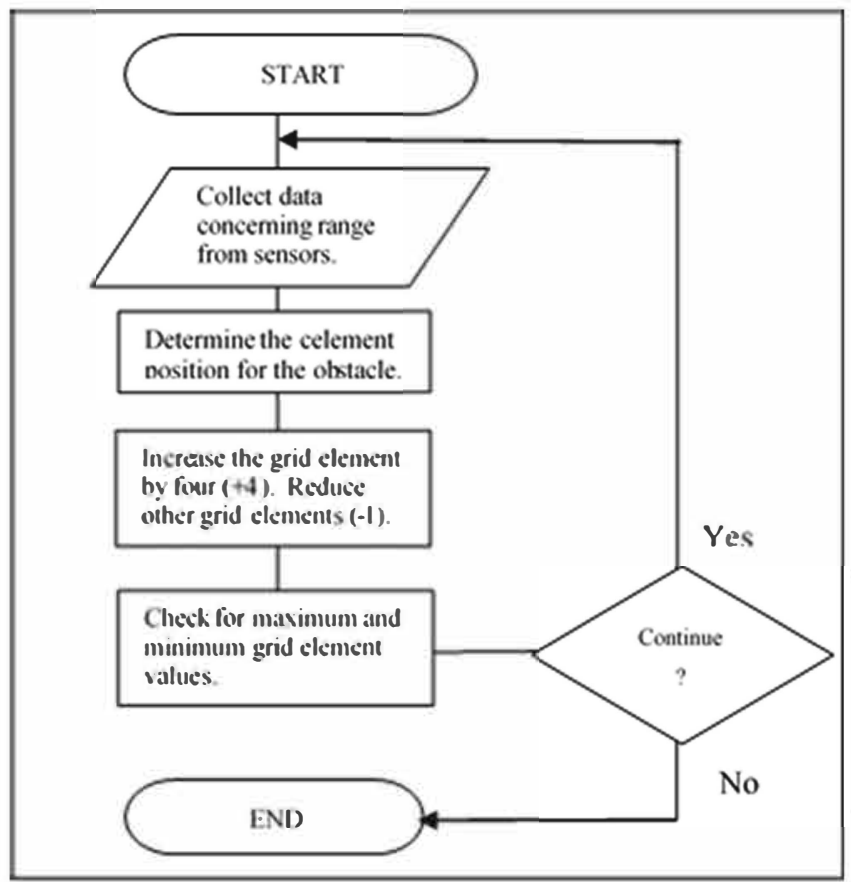

Fig. 3. Structure of the histogrammic object detection process.

Where JS1 and JS0 are Cartesian co-ordinates.

The sector occupied by the joystick was calculated using Magnitude and Angle. Position and confidence were denoted within an array of pairs of values:

- Joystick position for a desired speed was represented by "Magnitude".

- Confidence a joystick is within a sector was represented by "Angle Confidence".

If joysticks were held stationary then the grid element associated with their position increased and the other elements reduced. That element quickly increased in value. Shaking hands on the joystick increased other grid elements for a moment but they then reduced every time the system updated. If the joystick was moved to a new grid element then the new grid element increased and the previous grid element reduced.

Joystick position corresponded to a histogram and the histogram element with the largest value signified the preferred bearing. Figure 4 shows a joystick histogram. Position and angle of the joystick was tested by a module named JSArray. JSArray determined the zone inhabited by the joystick. The element corresponding to "angle confidence" (Aconf) increased by 40 . Other Aconf elements reduced by 20 . Histogram elements reduced in value rapidly but increased in value more gradually. 


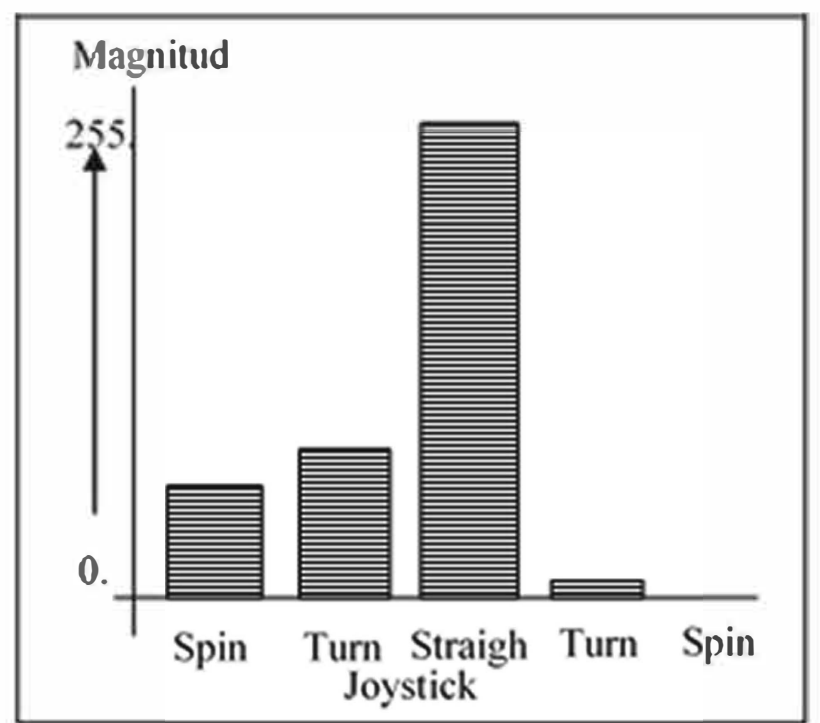

Fig. 4. Representation of joystick using histograms.

\section{Expert System}

Expert knowledge was provided by skilled wheelchair drivers and experienced rehabilitation engineers [37-44]. The system had to work in real time [45-50] in order to help disabled drivers. The two real-time inputs were from the sensor system and the joystick. Speed and direction were provided by the wheelchair driver and data about the environment and obstacles were provided by the sensors. A module called "Expert-Sensor" assessed sensor data and it suggested new bearings if required to avoid collisions. If data from the different sources disagreed then an expert called "FuzzMix" considered both inputs and decided on the outputs to be sent to the motor controllers. "JoyMon" deciphered what the wheelchair driver was trying to make the wheelchair do. The whole system was made up of: "GapOrDoor", "Expert-Sensor", "JoyMon", and "FuzzMix".

Control effort was distributed between the joystick and sensors by "FuzzMix". "FuzzMix" coordinated the sensor recommendation and joystick recommendation and any conflicts between them. "GapOrDoor", "JoyMon" and "Expert-Sensor" provided guidance. "Prox-Stop" was a failsafe function to stop the chair and "FuzzMix" could over-ride all the other inputs using "Prox-Stop". "FuzzMix" mixed joystick confidence and sensor data values. If joystick confidence was high then the position of the joystick was correctly reflecting the wishes of the wheelchair driver. In that case, the sensors had less effect. If confidence was low then it was necessary for the wheelchair to avoid an obstacle [46, 47].

Joystick consistency and position was checked by "JoyMon". If the joystick position was held steady then that established the wishes of the wheelchair driver. If the joystick was moving randomly then the driver was not in control or was unsure. In that case, the system depended more on the sensors for steering the chair. "Expert-Sensor" employed 
knowledge about the sensors. "Expert-Sensor" built a grid to contain data from the sensors and suggested potential maneuvers that would steer the wheelchair safely and elude collisions. "Expert-Sensor" didn't take the driver into account. "GapOrDoor" was the obstacle avoidance function. "GapOrDoor" used data from "Expert-Sensor" but could be overridden by "FuzzMix". Joystick data was merged with Sensor data so that:

$$
\begin{aligned}
& \text { Vout(right) }=\text { InJoy(right) }- \text { Dist(left) } \\
& \text { Vout(left) }=\text { InJoy (left) }- \text { Dist(right) }
\end{aligned}
$$

Where: InJoy was input joystick voltage, Vout was the controller output voltage, and Dist was the range to the nearest obstacle. InJoy, Vout and Dist were vectors having two values, right wheel and left wheel.

"GapOrDoor" could turn the wheelchair away from the nearest object, smoothly slow down the wheelchair as it gets close to an obstacle and center the wheelchair between objects, for example the surrounding frame of a doorway. "FuzzMix" allocated control to the joystick or the sensors subject to the environment, situation and desires of the wheelchair user. The relationships were:

- All joystick, No sensors.

- All sensors, No Joystick.

- Something in between.

"FuzzMix" assessed inputs and algorithms allocated control between joystick and sensors. Algorithms used distance-functions to create target values for left and right voltages. The distance-functions were:

$$
\begin{aligned}
& \text { RightTarg }=2.5 * \text { InstRange }[0]+100 ; \\
& \text { LeftTarg }=2.5 * \text { InstRange }[1]+100
\end{aligned}
$$

Where: InstRange[] $=$ instantaneous distance to obstacle.

Data from sensors was changed into a form matching Target (ADC) data. The position of the joystick was obtained from a joystick map divided into sectors: Forward, Stop, Reverse, Right Turn, Left Turn, Right Spin and Left Spin. A rule set within "ExpertSensor" was extracted from the mapping and used to search Sensor-Byte for objects so that "Expert-Sensor" could suggest action.

Software was downloaded to a micro-computer on the wheelchair. Software was written in a mix of low- and high-level languages. The programs were compiled and loaded into on board non-volatile memory. Systems were then tested in a variety of situations and environments. Algorithms were predictable and fast. If "Joystick" and "Expert-Sensor" signified "forward", then a bearing was set to drive straight ahead. Sensors continued to be quizzed to find distances to obstacles and speed was decreased if a wheelchair moved close to an obstacle. If joystick requested a "Turn" then a different algorithm was used. 


\section{Experiments}

Experiments involved driving wheelchairs through a variety of situations and environments. The response of the systems was acceptable and the wheelchair safely drove along corridors and aligned with the centers of door gaps with the controlling joystick held in a static (pushed forward) position. Paths of wheelchairs indicated that "Expert-Sensor" suggested suitable changes to speeds and directions. The algorithms successfully avoided obstacles.

The systems were not to replace wheelchair drivers but were intended to help them. Systems assisted wheelchair drivers in steering their chairs. Drivers swiftly learnt to drive. Sets of experimental runs took place to assess speed with and without assistance from sensors, using an existing system [3, 9] as a control check and then using the new systems described in this paper. Time taken to successfully finish experimental runs was recorded when a human driver was:

- Driving without any assistance,

- Assisted by existing previously published systems,

- Assisted by systems described in this paper.

Drivers drove their wheelchairs with the sensor systems helping them and researchers recorded times using digital laboratory clocks and stop watches. Experiments were to:

- Observe operation.

- Measure time taken by human drivers driving by themselves.

- Measure time taken with sensors and expert systems helping.

- Measure improvement.

- Capture suggestions and comments.

Experiments were conducted without any assistance. Then experiments were repeated with assistance (using the sensors). For each experiment, an obstacle course was set up and drivers had to handle the following:

LABORATORIES: Vertical walls. Objects on floor.

EMPTY CORRIDORS: Flat and sloping surfaces. Vertical walls. Door gaps. Obstacles in staggered arrangements.

COMPLICATED CORRIDORS: Flat and sloping surfaces. Vertical walls. Door gaps. Items on walls (e.g.: radiators). Obstacles offset. Door gaps.

OUTSIDE ENVIRONMENTS: More complicated environments with different flat and sloping surfaces. Vertical and sloping edges. Human beings present. Various objects.

Whenever possible, experiments were performed more than once. Wheelchair drivers could repeat experiments as often as they liked so that they learnt how the system(s) behaved and they could perform at their best.

Experiments were thought to be fun and were well-liked. Experiments encouraged some competition and wheelchair drivers tried to beat their best times and beat other wheelchair drivers. 
Experiments compared the speed of wheelchair drivers when being assisted and when driving by themselves in a variety of situations and environments. When a fast time was recorded then the driver made at least one more attempt at the other experiment (with/without sensors to assist) to check the result was not just because they had learnt more about how the systems worked.

\section{Results}

Figure 5 shows some results. The systems described in this paper were compared with existing systems [30,31]. Drivers driving with assistance were compared to the same driver driving without assistance. Average best time to complete various courses is shown on the vertical scale. Drivers driving without using any sensors to assist are on the left in Fig. 5. Drivers using the recently published systems $[3,30]$ are in the center and the improved systems described in this paper are on the right. The different testing routes are listed on the horizontal scale.

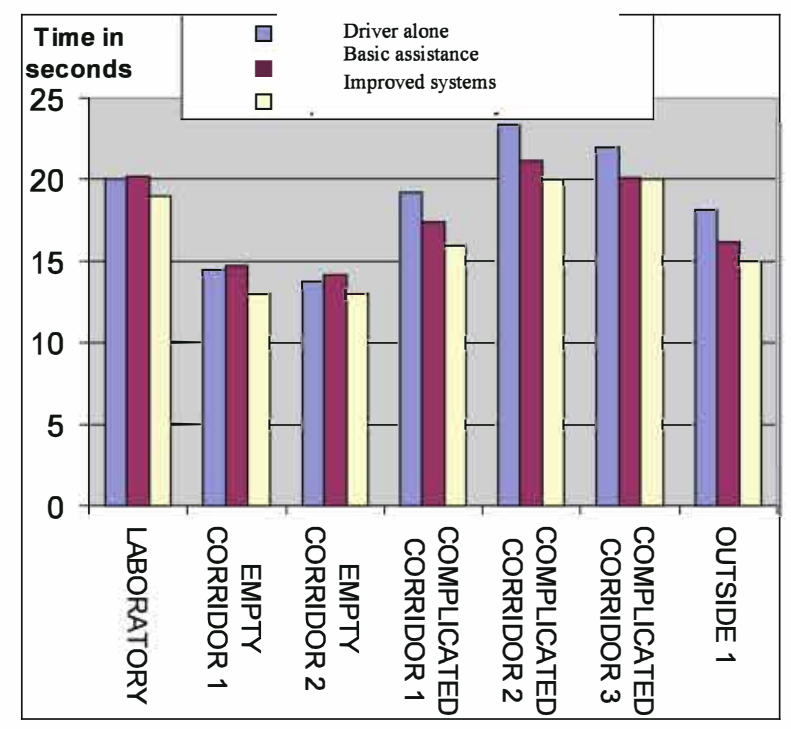

Fig. 5. Results from experiments.

On average, the new systems were quicker. In simpler empty corridors and laboratories, wheelchair drivers finished their driving tasks more quickly when they did not have any assistance form the sensors or computers. In more tricky outside and complicated corridors, wheelchair drivers completed their driving tasks more quickly with assistance from the sensors and computers. As gaps became narrower or environments became more complicated then wheelchair drivers took longer. Wheelchair drivers sometimes had to slow down or stop and reverse to avoid collisions. Drivers performed better with assistance in more complicated experiments. 
The new system presented in this paper reliably changed wheelchair directions and speeds and out-performed the recently published systems.

\section{Discussion and Conclusions}

Results from experiments were placed into two sets: with sensors assisting and without. Pairing removed a lot of randomness and variability. Results were statistically significant. Paired samples tests showed that driving was significantly different at $\mathrm{p}<0.05$ ( $95 \%$ probability that the result would not occur by chance). The methods and systems described here were significantly better than recently published systems. The new systems performed every test more quickly than recently published systems. Current work has now moved on to investigate new applications [51-55] and decision making [56-61].

Acknowledgment. Research in this paper was funded by EPSRC grant EP/S005927/1 and supported by The Chailey Heritage Foundation and the University of Portsmouth.

\section{References}

1. Kawaguchi, A., Noda, Y., Sato, Y., Kondo, Y., Terashima, K.: A mechatronics vision for smart wheelchairs. In: Proceedings of the 4th International Conference on Assistive Technologies, pp. 145-150, April 2008

2. Leaman, J., La, H.M.: A comprehensive review of smart wheelchairs: past, present, and future. IEEE Trans. Hum.-Mach. Syst. 47(4), 486-499 (2017)

3. Yukselir, M., Scarrow, K., Wilson, P., Cowan, T.: The brains behind the electric wheelchair, one of Canada's 'great artifacts'. The Globe and Mail, 27 August 2012

4. Carlson, T., Millan, J.D.R.: Brain-controlled wheelchairs: a robotic architecture. IEEE Robot. Auto. Mag. 20, 65-73 (2013)

5. Joshi, M.K., Gupta, M.V., Gosavi, M.M., Wagh, M.S.: A multifunctional smart wheelchair. Int. J. Adv. Res. Electron. Commun. Eng. 4(5), 1281-1284 (2015)

6. Sanders, D., Hudson, A.: A specific blackboard expert system to simulate and automate the design of high recirculation airlift reactors. Math. Comput. Simul. 53(1-2), 41-65 (2000)

7. Sanders, D., Okonor, O., Langner, M., et al.: Using a simple expert system to assist a powered wheelchair user. Advances in Intelligent Systems and Computing, vol. 1037, pp. 662-379. Springer, Heidelberg (2019)

8. Sanders, D.: Comparing speed to complete progressively more difficult mobile robot paths between human tele-operators and humans with sensor-systems to assist. Assem. Auto 29(3), 230-248 (2009)

9. Rahiman, M., Zakaria, Z., Rahim, R., et al.: Ultrasonic tomography imaging simulation of two-phase homogeneous flow. Sens. Rev. 29(3), 266-276 (2009)

10. Sanders, D.: Controlling the direction of "walkie" type forklifts and pallet jacks on sloping ground. Assem. Auto. 28(4), 317-324 (2008)

11. Sanders, D., Tewkesbury, G., Parchizadeh, H., et al.: Learning to drive with and without intelligent computer systems and sensors to assist. Advances Intelligent Systems Computing, vol. 868, pp. 1171-1181. Springer, Heidelberg (2019)

12. Sanders, D.A., Gegov, A., Haddad, M., Ikwan, F., Wiltshire, D., Tan, Y.C.: A rule-based expert system to decide on direction and speed of a powered wheelchair. In: Arai, K., Kapoor, S., Bhatia, R. (eds.) IntelliSys 2018. AISC, vol. 868, pp. 822-838. Springer, Cham (2019) 
13. Sanders, D.A., Gegov, A., Tewkesbury, G.E., Khusainov, R.: Sharing driving between a vehicle driver and a sensor system using trust-factors to set control gains. In: Arai, K., Kapoor, S., Bhatia, R. (eds.) IntelliSys 2018. AISC, vol. 868, pp. 1182-1195. Springer, Cham (2019)

14. Sanders, D.A.: Comparing ability to complete simple tele-operated rescue or maintenance mobile robot tasks with and without a sensor system. Sens. Rev. 30(1), 40-50 (2010)

15. Sanders, D.A.: Analysis of the effects of time delay on the tele-operation of a mobile robot in various modes of operation. Ind. Robot: Int. J. 36(6), 570-584 (2009)

16. Stott, I., Sanders, D.: New powered wheelchair systems for the rehabilitation of some severely disabled users. Int. J. Rehabil. Res. 23(3), 149-153 (2000)

17. Sanders, D., Stott, I.: A new prototype intelligent mobility system to assist powered wheelchair users. Ind. Rob. 26(6), 466-475 (1999)

18. Sanders, D.A., Baldwin, A.: "X-by-wire technology" Total Vehicle Technology: Challenging current thinking, pp. 3-12 (2001)

19. Sanders, D., Langner, M., Bausch, N., et al.: Improving human-machine interaction for a powered wheelchair driver by using variable-switches and sensors that reduce wheelchairveer. Adv. Intell. Syst. Comput. 1038, 1173-1191 (2019)

20. Sanders, D.A., Tewkesbury, G.E.: A pointer device for TFT display screens that determines position by detecting colours on the display using a colour sensor and an ANN. Displays 30(2), 84-96 (2009)

21. Sanders, D.A., Urwin-Wright, S.D., Tewkesbury, G.E., et al.: Pointer device for thin-film transistor and cathode ray tube computer screens. Electron. Lett. 41(16), 894-896 (2005)

22. Stott, I., Sanders, D.: The use of virtual reality to train powered wheelchair users and test new wheelchair systems. Int. J. Rehabil. Res. 23(4), 321-326 (2000)

23. Goodwin, M.J., Sanders, D.A., Poland, G.A., et al.: Navigational assistance for disabled wheelchair-users. J. Syst. Arch. 43(1-5), 73-79 (1997)

24. Sanders, D.A.: The modification of pre-planned manipulator paths to improve the gross motions associated with the pick-and-place task. Robotica 13(Part 1), 77-85 (1995)

25. Larsson, J., Broxvall, M., Saffiotti, A.: Laser-based corridor detection for reactive navigation. Ind. Rob.: Int'. J. 35(1), 69-79 (2008)

26. Lee, S.: Use of infrared light reflecting landmarks for localization. Ind. Rob. 36(2), 138-145 (2009)

27. Horn, O., Kreutner, M.: Smart wheelchair perception using odometry, ultrasound sensors, and camera. Robotica 27, 303-310 (2009)

28. Milanes, V., Naranjo, J.E., Gonzalez, C., et al.: Autonomous vehicle based in cooperative GPS and inertial systems. Robotica 26, 627-633 (2008)

29. Lim, D., Lee, S., Cho, D.: Design of an assisted GPS receiver and its performance analysis. In: IEEE Symposium on Circulation \& Systems, pp. 1742-1745 (2007)

30. Bloss, R.: Vision and robotics team up at the 2007 show. Ind. Rob.: Int'. J. 35(1), 19-26 (2008)

31. Sanders, D., Tan, Y., Rogers, I., Tewkesbury, G.: An expert system for automatic design-forassembly. Assem. Auto. 29(4), 378-388 (2009)

32. Sanders, D.: Environmental sensors and networks of sensors. Sens. Rev. 28(4), 273-274 (2008)

33. Hopper, D.: The long perspective for robotic vision. Assemb. Autom. 29(2), 122-126 (2009)

34. Sanders, D., Lambert, G., Graham-Jones, J., et al.: A robotic welding system using image processing techniques and a $\mathrm{CAD}$ model to provide information to a multi-intelligent decision module. Assem. Auto. 30(4), 323-332 (2010)

35. Sanders, D., Graham-Jones, J., Gegov, A.: Improving ability of tele-operators to complete progressively more difficult mobile robot paths using simple expert systems and ultrasonic sensors. Ind. Rob. Int. J. 37(5), 431-440 (2010) 
36. Sanders, D., Lambert, G., Pevy, L.: Pre-locating corners in images in order to improve the extraction of Fourier descriptors and subsequent recognition of shipbuilding parts. IMechE Part B 223(9), 1217-1223 (2009)

37. Sanders, D.: Progress in machine intelligence. Ind. Rob. 35(6), 485-487 (2008)

38. Sanders, D.: Recognizing shipbuilding parts using artificial neural networks and Fourier descriptors. Proc. Inst. Mech. Eng. Part B 223(3), 337-342 (2009)

39. Sanders, D., Stott, I.: Analysis of failure rates with a tele-operated mobile robot between a human tele-operator and a human with a sensor system to assist. Robotica 30(6), 973-988 (2012)

40. Sanders, D., Langner, M., Tewkesbury, G.: Improving wheelchair-driving using a sensor system to control wheelchair-veer and variable-switches as an alternative to digital-switches or joysticks. Ind. Rob.: Int. J. 37(2), 157-167 (2010)

41. Khatib, O.: Real-time obstacle avoidance for manipulators and mobile robots. In: Proceedings of IEEE International Conference on Robotics and Automation, vol. 5, no. 1, pp. 90-98 (1986)

42. Hudson, A., Sanders, D., Golding, H., et al.: Aspects of an expert design system for the wastewater treatment industry. J. Syst. Archit. 43(1-5), 59-65 (1997)

43. Sanders, D.: Introducing AI into MEMS can lead us to brain-computer interfaces and superhuman intelligence (invited viewpoint review paper) Assemb. Autom. 29(4), 309-312 (2009)

44. Sanders, D.: Ambient-intelligence, rapid-prototyping and where real people might fit into factories of the future. Assemb. Autom. 29(3), 205-208 (2009)

45. Sanders, D., Haynes, B., Tewkesbury, G., et al.: The addition of neural networks to the inner feedback path in order to improve on the use of pre-trained feed forward estimators. Math. Comput. Simul. 41(5-6), 461-472 (1996)

46. Sanders, D.: Perception in robotics. Ind. Rob. 26(2), 90-92 (1999)

47. Sanders, D.: System Specification 2. Microprocess. Microprogram. 38(1-5), 833-833 (1993)

48. Sanders, D., Harris, P., Mazharsolook, E.: Image modelling in real-time using spheres and simple polyhedra. In: 4th International Conference on Image Processing and Its Applications, vol. 354, pp. 433-436 (1992)

49. Sanders, D., Hudson, A., Tewkesbury, G.: Automating the design of high-recirculation airlift reactors using a blackboard framework. Expert Syst. Appl. 18(3), 231-245 (2000)

50. Sanders, D.: Real time geometric modelling using models in an actuator space and Cartesain space. J. Robot. Syst. 12(1), 19-28 (1995)

51. Fahimi, F., Nataraj, C., Ashrafiuon, H.: Real-time obstacle avoidance for multiple mobile robots. Robotica 27, 189-198 (2009)

52. Tewkesbury, G., Sanders, D.: The automatic programming of production machinery for deflashing plastic parts. In: Advances in Manufacturing Technology VIII, pp. 279-283 (1994)

53. Tewkesbury, G., Sanders, D.: The use of distributed intelligence within advanced production machinery for design applications. In: Total Vehicle Technology: Challenging Current Thinking, pp. 255-262 (2001)

54. Sanders, D.A., Robinson, D.C., Hassan, M., Haddad, M., Gegov, A., Ahmed, N.: Making decisions about saving energy in compressed air systems using ambient intelligence and artificial intelligence. In: Arai, K., Kapoor, S., Bhatia, R. (eds.) IntelliSys 2018. AISC, vol. 869, pp. 1229-1236. Springer, Cham (2019)

55. Sanders, D., Wang, Q., Bausch, N., Huang, Ya., Khaustov, S., Popov, I.: A method to produce minimal real time geometric representations of moving obstacles. In: Arai, K., Kapoor, S., Bhatia, R. (eds.) IntelliSys 2018. AISC, vol. 868, pp. 881-892. Springer, Cham (2019)

56. Haddad, M., Sanders, D., Gegov, A., et al.: Combining multiple criteria decision making with vector manipulation to decide on the direction for a powered wheelchair. In: Advances Intelligent Systems Computing, vol. 1037, pp. 680-693. Springer, Heidelberg (2019)

57. Haddad, M., Sanders, D., Bausch, N.: Selecting a robust decision making method to evaluate employee performance. Int. J. Manag. Decis. Making 18(4), 333-351 (2019) 
58. Haddad, M., Sanders, D., Tewkesbury, G.: Selecting a discrete multiple criteria decision making method to decide on a corporate relocation. Arch. Bus. Res. 7(5), 48-67 (2019)

59. Haddad, M., Sanders, D.: Selecting a best compromise direction for a powered wheelchair using PROMETHEE. IEEE Trans. Neural Syst. Rehabil. Eng. 27(2), 228-235 (2019)

60. Haddad, M., Sanders, D., Tewkesbury, G., et al.: Initial results from using preference ranking organization METHods for enrichment of evaluations to help steer a powered wheelchair. Adv. Intell. Syst. Comput. 1037, 648-661 (2019)

61. Haddad, M., Sanders, D., Bausch, N., Tewkesbury, G., Gegov, A., Hassan, M.: Learning to make intelligent decisions using an expert system for the intelligent selection of either PROMETHEE II or the analytical hierarchy process. In: Arai, K., Kapoor, S., Bhatia, R. (eds.) IntelliSys 2018. AISC, vol. 868, pp. 1303-1316. Springer, Cham (2019) 